\title{
Health Disparities Between Appalachian and Non-Appalachian Counties in Virginia USA
}

\author{
Elizabeth L. McGarvey • MaGuadalupe Leon-Verdin • \\ Lydia F. Killos • Thomas Guterbock • \\ Wendy F. Cohn
}

Published online: 23 September 2010

(c) The Author(s) 2010. This article is published with open access at Springerlink.com

\begin{abstract}
The examination of health disparities among people within Appalachian counties compared to people living in other counties is needed to find ways to strategically target improvements in community health in the United States of America (USA). Methods: A telephone survey of a random sample of adults living in households within communities of all counties of the state of Virginia (VA) in the USA was conducted. Findings: Health status was poorer among those in communities within Appalachian counties in VA and health insurance did not make a difference. Health perception was significantly worse in residents within communities in Appalachian counties compared to non-Appalachian community residents ( 30.5 vs. $17.4 \%$ rated their health status as poor/fair), and was worse even among those with no chronic diseases. Within communities in Appalachian counties, black residents report significantly better health perception than do white
\end{abstract}

E. L. McGarvey $(\bowtie) \cdot$ M. Leon-Verdin · W. F. Cohn

School of Medicine, Department of Public Health Sciences,

University of Virginia, P.O. Box 800717,

Charlottesville, VA 22908, USA

e-mail: rel8s@virginia.edu

M. Leon-Verdin

e-mail: lupitalv2001@gmail.com

W. F. Cohn

e-mail: wfc2r@virginia.edu

L. F. Killos

Office of Health Promotion, University of Virginia,

P.O. Box 00760, Charlottesville, VA 22908, USA

e-mail: 1fk4y@virginia.edu

T. Guterbock

Department of Sociology \& Cooper Center for Survey Research,

University of Virginia, Charlottesville, VA 22908, USA

e-mail: tmg1p@virginia.edu residents. Conclusion: Residents living in communities in Appalachian counties in VA are not receiving adequate health care, even among those with health insurance. More research with a larger ethnic minority sample is needed to investigate the racial/ethnic disparities in self-reported health and health care utilization within communities.

Keywords Appalachia - Ethnicity $\cdot$ Health care utilization - Health perceptions · Health status . Poverty · USA

The Appalachian mountain range extends across 13 states in the United States (US) from New York to Mississippi, including 420 counties, forming three regions: Northern, Central and Southern Appalachia [1]. All are characterized to varying degrees by mountains, hollows and rivers that wind along mostly low-density populated areas, with about two of three counties considered rural [2]. In Appalachian counties in Virginia (VA), the dominant culture and nuances of the language of the people are historically linked to those who migrated from northwestern European countries (e.g. Scotland, Ireland, Wales, England) [3-5]. A small minority of Jewish, Mediterranean, Middle Eastern and other ethnic groups (e.g. Melungeons) are represented [6-11].

There are continuing debates about what qualities best define Appalachian culture. Positive core values reported include: strong sense of community, strong family support systems and social ties, religious affiliation, pride in self and family, independent self-reliance, the importance of justice, loyalty, religion, faith in God, strong work ethic, trustworthiness, and a feeling of belonging in the mountains [12-14]. More problematic stereotypes and/or cultural norms are also reported, such as: fatalistic outlook, distrust 
of outsiders, and distrust of formalized medical systems [15-17]. However, it is a veritable fact, based upon quantitative data, that socio-economic indicators are much worse for the communities in the Appalachian region compared to those in the rest of the United States.

The Appalachia Regional Commission reports that per capita personal income, real wages, salaries per job, and market income are significantly lower in all communities in Appalachian areas, compared to the respective state's averages. Common are 3-year unemployment rates $125 \%$ above the national average, poverty rates of $125 \%$ above the national average or higher and 67\% lower income. Central Appalachia, where most counties in VA are located, has the worst economic indicators of all the Appalachian regions [1]. Health disparities, many of which are preventable, have been found to be related to both social class (i.e. income, occupation, educational attainment) and region [18-21]. People who live in communities in rural locations have been found to be less likely to seek medical care or receive specialized treatments [22]. Most of the counties and towns in Appalachia in Virginia are rural with relatively small counties.

More than 5\% of the residents in Appalachian counties and cities in VA are black [23]. Residents of Appalachian counties were found to have significantly higher rates of poverty and lower rates of high school and college graduation, but little is known of race/ethnic differences in health disparities [1, 24, 25]. Appalachian counties in VA have higher rates of premature mortality than the rest of the state, with some counties in Appalachian communities showing twice the death rate per 100,000 residents [26]. Furthermore, death from cancer, heart disease, diseases of the lung and stroke have been found at significantly higher than national rates in Appalachia [1, 18, 27-29]. VA residents in communities within some Appalachian counties are "23\% more likely to die from heart disease; $28 \%$ more likely to die from diabetes; and are $44 \%$ more likely to die from chronic obstructive pulmonary disease" when compared with residents in the rest of the state [29]. More evidence-based research is needed to explore more definitively why this is so using information obtained from the residents in the communities in addition to official mortality and morbidity statistics.

In a number of communities in Appalachia, lack of health care resources have been associated with the lack of specialty care providers in the area and unavailable times for appointments, at times up to 3 months for outpatient visits [25]. Health care utilization in this region is low even when it is available, possibly due to lack of insurance or insufficient funds for insurance co-payments as well as barriers related to Appalachian culture [25]. Lack of cultural competence on the part of available health care providers has been reported as one barrier to seeking care [30].
In Appalachian counties, community residents in one survey were more likely to self-report their health status as "poor" than were those living outside the area, but how their perception was related to health care utilization is not known [31]. Health status, which is often measured on scales from "poor" to "excellent" can be defined as all aspects of health that relate to one's daily living $[32,33]$. Poorer health status has been shown to be associated with higher numbers of chronic conditions and disease diagnoses, but whether this is accurate for Appalachia compared to non-Appalachia communities in VA has not been reported to date [34]. Health perception and health status, which are related to health care utilization, have been found to differ between black and white participants in some studies, but these comparisons have not previously been reported in Virginia Appalachian compared to nonAppalachian communties [35].

Health perception or perceived health (i.e. a person's perception of his or her own health status) can be a mediator between critical thinking and health behaviors, which is important in preventive medicine as well as health care utilization [36, 37]. For example, a person may be aware that obesity is associated with a number of health risks, but may only decide on a plan to increase physical activity or improve eating habits when that person perceives his/her health status as "poor" due to a diagnosis of diabetes and acute pain. There is a need to compare health perceptions and health care utilization between Appalachian and other counties in VA since current information on the link between getting care associated with one's own perception of health has not been reported. In addition, race/ethnic differences within and between Appalachian and other counties in VA need to be examined to better inform strategically targeted public health practices related to place and race/ethnicity within communities. This study investigates health status and health perception between residents in communities in Appalachian counties and counties in the rest of VA. These factors have been associated with health behavior change, health care utilization, and are important in health policy development related to reducing the premature morality rates $[26,38-42]$.

Health behaviors and risk perceptions vary by socioeconomic status (SES) with lower SES individuals less likely to participate in preventing health problems [36]. For example, residents in rural areas who have both low incomes and levels of education tend not to participate in positive health behavior change, such as smoking cessation, compared with their neighbors who have both higher incomes and education [43]. Since 70-90\% of the management of illness is done by individuals who have no medical training (i.e. the lay public) [44-46], the provision of health care and health behavior change could be enhanced immeasurably by understanding the lay public's 
health perceptions and behaviors within the context of community, culture and race/ethnicity as well as socioeconomic status and the availability of health insurance [44].

The purpose of this study is to investigate the health perceptions of community residents in Appalachian counties related to their health status and health care utilization, compared to those who do not live in the Appalachian counties of the state. Specifically, we investigated: (1) Differences in health perception, health status and health care utilization between residents in communities within VA counties in Appalachia and those in other counties in the state and (2) Race/ethnic differences within counties in Appalachia and other counties in the state with regard to health perception, health status, and health care utilization.

\section{Methods}

\section{Procedures}

A telephone survey of a random sample of households from all counties in VA was conducted [45]. The survey protocol was approved by the Institutional Review Board for the Social and Behavioral Sciences at the University of Virginia (UVa). Inclusion criteria included anyone over the age of 18 who had a land-line telephone and agreed to participate. No personal identifying information was elicited from the respondents. All information was voluntarily obtained from individually selected respondents, chosen at random, to avoid possible bias related to characteristics of people who might be more likely to answer the telephone (e.g. people who did not work) [47]. Respondents were free to hang up at any time during the interview. The telephone interviews were conducted by trained interviewers from the UVa Center for Survey Research using Computer Assisted Telephone Interviewing (CATI) software and entered into SPSS 16 for data cleaning, error checking and analysis.

\section{Description of the Sample}

The primary sample for the study was a statewide, listassisted Random Digit Dial sample, which was matched to directory listings in order to obtain addresses where possible. This sample was supplemented with two smaller targeted directory-listed samples: one drawn from census tracts having $35 \%$ or more black residents and one from census tracts with median household incomes less than $\$ 35,000$. The use of these subsamples helped to ensure sample representativeness with respect to race/ethnicity and income. Advance letters were mailed to households whose addresses were known; telephone numbers without known addresses were also attempted. The final sample of completed telephone interviews included 1,201 respondents age 18 or older. Phone interviews averaged 29 minutes in length. Cooperation rates were $47.6 \%$, resulting in a margin of error of $\pm 2.8 \%$, while an overall response rate (i.e. someone answered the telephone, but refused to participate in the survey) was $22 \%$, which is an acceptable standard for telephone surveys of this type [48]. Missing data on zip code, education level, age and health insurance status excluded a number of cases for the present analyses, resulting in a final sample size of 1,128 VA residents from all counties of the state.

\section{Instrument}

The questionnaire used in the telephone survey was developed by an expert panel of social scientists at UVa who reviewed and selected the most appropriate items from other valid and reliable instruments. The survey instrument was pilot tested and refined prior to use [45]. This study used information from seven socio-demographic items (e.g. zip code location within the state, age, sex, race/ethnicity, education level, having current health insurance). Eight health-related items from the questionnaire were also used in this study. Personal health status included having a current disease, as indicated by self-report of four chronic conditions. Health perception included one overall selfappraisal item ("In general, how would you rate your own health?"). Health care utilization was obtained from three additional items regarding the number of times in the last 12 months that the respondent went to (a) "an emergency room", (b) "a doctor's office or clinic", or (c) "was a patient in a hospital overnight or longer".

\section{Statistical Analysis}

Individual respondent data were recoded according to zip code information into the Appalachian counties, versus all other counties in VA. To ensure highest statistical power for the analyses only missing zip code cases were excluded ( $n=1,164)$. Descriptive statistics and chi-square analyses were calculated to compare differences by categorical and independent factors. Current health status was analyzed using a logistic model, adjusting for race. Perception of the respondent's own health (1) and health care utilization (2) were compared between VA counties in Appalachia and respondents in counties outside of Appalachia, adjusting for diagnosis of a chronic disease, education level, insurance, and race/ethnicity, using a General Linear Model (GLM) in 1 and a multivariable GLM in 2. Perception of health (PH) was categorized as (1) poor, (2) fair, (3) good, and (4) excellent. The race/ethnicity variable was categorized as (1) white, (2) black and (3) others, which included 
Asian, American Indian and other ethnicities (e.g. Hispanic). Having insurance was recoded to a yes/no variable by considering any type of private or public insurance (e.g. Medicaid) as having insurance and considering reports of no insurance, paying out of pocket, going to a free clinic, paying on a sliding scale as having no insurance. Having a chronic disease was a yes/no response based on individual report of a diagnosis of cancer, diabetes, heart disease, and high blood pressure. Education level is categorized by nine levels, listed in Table 1. Age was excluded from the model for two reasons: first it is highly correlated with having a disease $(r=.5, P<.001)$, second, there was no significant difference in age between the Appalachian area and the rest of the state. Household income was also excluded from the analysis due to a high number of missing cases and refusals to respond. Furthermore, household income was highly correlated with education level $(r=.5, P<.001)$. The relationship between health perception and health care utilization was analyzed using GLM, adjusted for having chronic diseases and region.

\section{Results}

\section{Participants}

Demographic information for the Appalachian counties in VA and the other counties in VA are listed in Table 1. There are no significant differences in age, sex, and having insurance but differences in race/ethnicity, education level, and income are consistent with government statistics [24].

\section{Current Health Status}

Respondents in Appalachian counties, adjusting for race/ ethnicity, report significantly higher rates of cancer
Table 1 Characteristics of Appalachian county residents compared to all other counties residents in Virginia

\begin{tabular}{|c|c|c|c|c|}
\hline & $\begin{array}{l}\text { Appalachian residents } \\
N=158(100 \%)\end{array}$ & $\begin{array}{l}\text { Non Appalachian residents } \\
N=970(100 \%)\end{array}$ & Statistics $(d f)$ & $P$-value \\
\hline Age $($ Mean \pm SD) & $54.83 \pm 17.6$ & $54.29 \pm 17.1$ & $t(1126)=.37$ & NS \\
\hline Sex & & & $\chi^{2}(1)=.015$ & NS \\
\hline Male & $64(40.5)$ & $388(40.0)$ & & \\
\hline Female & $94(59.5)$ & $582(60.0)$ & & \\
\hline Race/ethnicity & & & $\chi^{2}(2)=11.1$ & .004 \\
\hline White & $143(90.5)$ & $776(80.0)$ & & \\
\hline Black & $9(5.7)$ & $149(15.4)$ & & \\
\hline Others & $6(3.8)$ & $45(4.6)$ & & \\
\hline Education & & & $\chi^{2}(8)=45.9$ & $<.001$ \\
\hline Less than 9th grade & $10(6.3)$ & $19(2.0)$ & & \\
\hline 9th to 12 th, did not grad & $11(7.0)$ & $65(6.7)$ & & \\
\hline High School graduate & $49(31.0)$ & $216(22.3)$ & & \\
\hline Some college & $31(19.6)$ & $201(20.7)$ & & \\
\hline 2 year degree $\mathrm{AA} / \mathrm{AS}$ & $29(18.4)$ & $87(9.0)$ & & \\
\hline 4 year degree BA/BS & $13(8.2)$ & $187(19.3)$ & & \\
\hline Some graduate work & $6(3.8)$ & $40(4.1)$ & & \\
\hline Masters/professional & $8(5.1)$ & $118(12.2)$ & & \\
\hline Doctorate level & $1(.6)$ & $37(3.8)$ & & \\
\hline Have insurance & & & $\chi^{2}(1)=.351$ & NS \\
\hline Yes & $140(88.6)$ & $843(86.9)$ & & \\
\hline No & $18(11.4)$ & $127(13.1)$ & & \\
\hline Income & $N=143$ & $N=836$ & $\chi^{2}(6)=38.0$ & $<.001$ \\
\hline$\$ 14,999$ or less & $25(17.5)$ & $78(9.3)$ & & \\
\hline$\$ 15,000-\$ 34,999$ & $47(32.9)$ & $171(20.5)$ & & \\
\hline$\$ 35,000-\$ 49,999$ & $27(18.9)$ & $135(16.1)$ & & \\
\hline$\$ 50,000-\$ 74,999$ & $28(19.6)$ & $186(22.2)$ & & \\
\hline$\$ 75,000-\$ 99,999$ & $11(7.7)$ & $100(12.0)$ & & \\
\hline$\$ 100,000-\$ 149,999$ & $4(2.8)$ & 99 (11.8) & & \\
\hline$\$ 150,000$ or more & $1(.7)$ & $67(8.0)$ & & \\
\hline
\end{tabular}


$(\mathrm{OR}=1.58, P=.04)$. However, no significant differences were found in respondents with a current diagnosis of diabetes $(\mathrm{OR}=.751, P=.34)$, heart disease $(\mathrm{OR}=1.17$, $P=.63)$ or high blood pressure $(\mathrm{OR}=.939, P=.72)$. There were more white than black respondents who were told they have cancer $(\mathrm{OR}=.54, P=.04)$ and heart disease $(\mathrm{OR}=.95, P=.83)$. More black respondents were told that they have diabetes $(\mathrm{OR}=1.68, P=.03)$ and high blood pressure $(\mathrm{OR}=2.14, P<.001)$ than white respondents. Number of people and the percentages are reported in Table 2 . The probability of having a chronic disease was not significantly higher in residents who live in Appalachian counties $(\mathrm{OR}=1.13, P=.47)$ but was higher for black residents overall $(\mathrm{OR}=1.53, P=.015)$.

Having a Serious Health Problem and Perception of General Health

Overall, respondents in Appalachian counties (55.5\%) were not significantly more likely to report having a chronic disease than were respondents in other counties $(53.3 \%)$ $\left(\chi^{2}(1)=.271, P=.603\right)$. Among those who reported having chronic diseases, there was no significant difference in the number of chronic diseases reported by Appalachian versus other counties $\left(t\right.$-test $\left._{(622)}=-.29, P=.78\right)$. The Appalachian counties average is $1.48(\mathrm{SD}=.77)$ chronic diseases while other counties average is $1.51(\mathrm{SD}=.70)$.
There were no significant differences in the number of chronic diseases per person $\left(\chi^{2}(4)=3.5, \quad P=.47\right)$. Respondents in Appalachian counties who reported having a chronic disease perceived their general health as significantly worse than those in other counties who had chronic diseases. Among those with no cancer, heart disease, diabetes or high blood pressure, respondents in Appalachian counties (12.4\%) were almost twice as likely to report their general health as "poor or fair" compared to those in other counties $(7.7 \%)\left(\chi^{2}(3)=17.09, P=.01\right)$. Percentages and frequencies of the perception of health are shown in Table 3.

\section{Health Perception}

After controlling for a baseline group's differences, one's perception of his or her own health $(\mathrm{PH})$, ranging from excellent to poor, tends to be worse in individuals who have chronic diseases $\left(F\right.$-test $\left._{(1,1130)}=132.72, P<.001\right)$. The PH increases as level of education increases $\left(F\right.$-test $\left._{(8,1130)}=6.66, P<.001\right)$. Individuals with insurance report no higher $\mathrm{PH}$ than respondents without it $\left(F\right.$-test $\left._{(1,1130)}=.007, P=.935\right)$. There was no significant difference by race/ethnicity $\left(F\right.$-tests $_{(1,1130)}=.648$, $P=.523)$; although white respondents $(83.5 \%)$ in other counties reported higher rates of good or excellent PH compared to the other respondents (see Table 2).

Table 2 Frequencies and percentage of people who reported having a chronic disease, their health perception and use of the health care utilities in residents of Virginia, $(N=1,164)$

\begin{tabular}{|c|c|c|c|c|}
\hline & \multicolumn{2}{|l|}{ Appalachian counties } & \multicolumn{2}{|c|}{ Other counties in Virginia } \\
\hline & White residents $n(\%)$ & Black residents $n(\%)$ & White residents $n(\%)$ & Black residents $n(\%)$ \\
\hline \multicolumn{5}{|l|}{ Current health status } \\
\hline Cancer & $30(20.3)$ & $0(0)$ & $105(13.3)$ & $13(8.3)$ \\
\hline Diabetes & $10(6.8)$ & $1(11.1)$ & $89(11.3)$ & $27(17.3)$ \\
\hline Heart disease & $23(15.5)$ & $1(11.1)$ & $121(15.3)$ & $23(14.7)$ \\
\hline High blood pressure & $57(38.5)$ & $3(33.3)$ & $311(39.4)$ & $93(59.6)$ \\
\hline \multicolumn{5}{|l|}{ Number of diseases } \\
\hline No diseases & $65(43.9)$ & $6(66.7)$ & $375(47.5)$ & $55(35.3)$ \\
\hline 1 Chronic disease & $56(37.8)$ & $2(22.2)$ & $247(31.3)$ & $58(37.2)$ \\
\hline 2 Chronic diseases & $19(12.8)$ & $0(0)$ & $127(16.1)$ & $33(21.2)$ \\
\hline More than 2 & $8(5.5)$ & $1(11.1)$ & $40(5.0)$ & $10(6.4)$ \\
\hline \multicolumn{5}{|l|}{ Perception of health } \\
\hline Excellent & $19(12.8)$ & $1(11.1)$ & 194 (24.6) & $29(18.6)$ \\
\hline Good & $84(56.8)$ & $6(66.7)$ & $465(58.9)$ & $78(50.0)$ \\
\hline Fair & $37(25.5)$ & $1(11.1)$ & $96(12.2)$ & $42(26.9)$ \\
\hline Poor & $8(5.4)$ & $1(11.1)$ & $34(4.3)$ & $7(4.5)$ \\
\hline \multicolumn{5}{|l|}{ Health care utility } \\
\hline Outpatient visit & $129(87.2)$ & $6(66.6)$ & $694(88.0)$ & $135(86.5)$ \\
\hline Emergency room & $32(21.6)$ & $4(44.4)$ & $146(18.5)$ & $51(32.9)$ \\
\hline Inpatient Hospital & $22(14.9)$ & $1(11.1)$ & $89(11.3)$ & $23(14.7)$ \\
\hline
\end{tabular}


Table 3 Frequencies in perception of health stratified by place of living and having chronic diseases in resident of Virginia, $(N=1,164)$

\begin{tabular}{|c|c|c|c|c|}
\hline & \multicolumn{2}{|l|}{ Appalachian counties } & \multicolumn{2}{|c|}{ Other counties in Virginia } \\
\hline & $\begin{array}{l}\text { Do not have chronic } \\
\text { diseases } n(\%)\end{array}$ & $\begin{array}{l}\text { Have chronic } \\
\text { diseases } n(\%)\end{array}$ & $\begin{array}{l}\text { Do not have chronic } \\
\text { diseases } n(\%)\end{array}$ & $\begin{array}{l}\text { Have chronic } \\
\text { diseases } n(\%)\end{array}$ \\
\hline Excellent & $10(13.7)$ & $10(11.0)$ & $179(38.3)$ & $65(12.2)$ \\
\hline Good & $54(74.0)$ & $40(44.0)$ & $252(54.0)$ & $315(59.1)$ \\
\hline Fair & $8(22.0)$ & $33(36.3)$ & $31(6.6)$ & $114(21.4)$ \\
\hline Poor & $1(2.4)$ & $8(8.8)$ & $5(1.1)$ & $39(7.3)$ \\
\hline
\end{tabular}

In Appalachian counties, more black respondents reported good or excellent PH compared to black respondents in other counties. More respondents from the other race/ethnic group had good and excellent $\mathrm{PH}$ in other counties than did those in Appalachian counties. Differences in health perception between respondents in Appalachian counties and other counties were statistically significant $\left(F\right.$-tests $\left._{(1,1130)}=10.17, P=.001\right)$.

\section{Health Care Utilization in the Past Year}

Over $87 \%$ of the respondents reported visiting a doctor's office or clinic, over $21 \%$ of the respondents reported using the ER for health care, and over $12 \%$ of respondents reported at least one overnight hospital stay in the last year. Health care utilization depends on education level (Pillai's Trace $=$ $2.11, P<.001$ ), having insurance (Pillai's Trace $=5.10$, $P=.002$ ), race/ethnicity (Pillai's Trace $=3.17, P=.004$ ), having chronic diseases (Pillai'sTrace $=29.27, P<.001$ but not by living in Appalachian or other counties (Pillai's Trace $=.218, P=.884$ ). There were no significant differences in the number of respondents who visited the doctor's office or clinic for care in Appalachian counties (86.6\%) compared to those in other counties $(87.3 \%)$, who used the ER for health care (22.6 vs. $21.0 \%$ ) or who reported overnight hospitalization (14.0 vs. $11.9 \%$ ) in the past year. There were no significant differences among race/ethnicities with regard to outpatient care within the past year, but black respondents reported greater use of the ER for health care (44 and 33\% in Appalachian and other counties) compared to white respondents (22 and 19\%, respectively (see Table 2). Between subject effects are summarized in Table 4 for each of the variables that measured the health care utilization.

\section{Health Care Utilization and Perception of General Health $(\mathrm{PH})$}

There were differences in perception of health by health care utilization adjusting for residing in Appalachian or other counties and whether or not the respondent had a chronic disease. In general, health perception was worse in residents of Appalachian counties and among those who have chronic diseases (Table 5). Most of the respondents
Table $4 F$-Statistics and $P$-values for covariates of the health care utilization in residents of Virginia

\begin{tabular}{lrrr}
\hline & $F$-Statistic & $d f$ & $P$-value \\
\hline Outpatient care & & & \\
Have chronic disease & 74.644 & 1,1130 & $<.001$ \\
Education level & 1.780 & 8,1130 & .077 \\
Have insurance & 13.215 & 1,1130 & $<.001$ \\
Race/ethnicity & .239 & 2,1130 & .788 \\
Region & .088 & 1,1130 & .767 \\
Emergency room & & & \\
Have chronic disease & 7.087 & 1,1130 & .008 \\
Education level & 2.844 & 8,1130 & .004 \\
Have insurance & .933 & 1,1130 & .334 \\
Race/ethnicity & 7.938 & 2,1130 & $<.001$ \\
Region & .391 & 1,1130 & .532 \\
Inpatient hospital stay & & & \\
Have chronic disease & 20.266 & 1,1130 & $<.001$ \\
Education level & 2.158 & 8,1130 & .028 \\
Have insurance & .183 & 1,1130 & .669 \\
Race/ethnicity & .216 & 2,1130 & .806 \\
Region & .014 & 1,1130 & .904 \\
\hline
\end{tabular}

who visit the ER $\left(F_{(1,1160)}=68.7, P<.001\right)$ and those who were hospitalized overnight $\left(F_{(1,1160)}=35.2\right.$, $P<.001)$, have worse perception of their own health.

\section{Limitations}

The information obtained in this random telephone survey has certain limitations. Only those over the age of 18 with a land telephone line were included in the study. As such, those who exclusively use cellular telephones and those without phones were excluded from the study. Our overall sample averaged age 54 , while the state median age is about 36 years [23]. While efforts were made to oversample for black residents the numbers who are represented from Appalachian counties is relatively small, suggesting larger numbers are needed to better investigate race/ethnic differences. Furthermore, about $20 \%$ of those in VA Appalachia are known not to have health insurance; our respondents were more likely to have health insurance, perhaps due to higher incomes, which is suggested by the 
Table 5 Statistics for the analyses of health perception and health care utilization in residents of Virginia

\begin{tabular}{lrrr}
\hline & $B$ & $t$-Statistic & $P$-value \\
\hline Outpatient care & & & \\
Intercept & 2.237 & 75.464 & $<.001$ \\
No chronic diseases & -.503 & -11.847 & $<.001$ \\
Appalachia region & .239 & 4.026 & $<.001$ \\
No visit to the doctor office & -.102 & -1.607 & .108 \\
Emergency room & & & \\
Intercept & 2.535 & 54.498 & $<.001$ \\
No chronic diseases & -.482 & -11.910 & $<.001$ \\
Appalachia region & .233 & 4.039 & $<.001$ \\
No visit to ER & -.408 & -8.287 & $<.001$ \\
Inpatient hospital stay & & & \\
Intercept & 2.542 & 42.447 & $<.001$ \\
No chronic diseases & -.481 & -11.646 & $<.001$ \\
Appalachia region & .230 & 3.942 & $<.001$ \\
No inpatient hospital & -.373 & -5.935 & $<.001$ \\
\hline
\end{tabular}

fact that they had telephones. This limits the ability to generalize the findings beyond those who have similar demographic and ethnic characteristics. Finally, it can be assumed that only those who have fewer barriers in talking to strangers about personal health issues on the telephone were open to being interviewed, and residents who may be most representative of Appalachian culture (e.g. reluctant to talk about family health issues, distrust of outsiders, etc.) may be less represented in the sample.

\section{Discussion}

Appalachian counties in VA are known to represent a health disparity region for residents within those communities due to the significantly higher morbidity and mortality rates compared to the residents in communities within other counties in Virginia [1, 24, 49]. With regard to health status, this study found significantly higher rates of residents in Appalachian counties self-reported a cancer diagnosis. This finding is consistent with higher incidence of specific cancers in Appalachia reported in other states [27]. However, in this study, county residents in communities in Appalachian counties were not more likely to selfreport being diagnosed with diabetes and heart disease although the mortality rates for these diseases, as well as cancer, have been significantly higher in the last decade. This suggests that the increased risk of mortality, such as those reported where the people are " $23 \%$ more likely to die from heart disease and $28 \%$ more likely to die from diabetes" may be related to late stage diagnosis and less participation in public health interventions (e.g. early detection screenings.) within those communities [29] This may suggest that there are more successful efforts being made to provide early detection for cancer services than for other diseases in those counties' communities. Since there are currently higher mortality rates for cancer reported in Appalachia in VA and there are also higher rates of diagnoses found in this study that may lead to reduction in future mortality rates for cancer, this finding may be encouraging.

Overall, this study did not find significant differences in the number of chronic diseases per person by place of residence. Nor did it find that poorer health status was associated with higher number of chronic diseases in residents of communities within Appalachian counties. This may be due to more residents having undiagnosed chronic diseases due to lack of access to health care or preventive services.

Among those residents who reported having serious diseases (e.g. cancer), significantly more residents of communities within Appalachian counties regarded their overall general health as "poor or fair" than did residents within communities of other counties. It may be that community residents of Appalachian counties wait longer to seek health care than do those from other counties due to a cultural tendency to be "self-reliant", and perhaps "fatalistic", which may result in cancer and other conditions being diagnosed and treated at later stages. The fact that this difference remained after controlling for health insurance suggests this. Furthermore, other barriers to adequately managing these conditions over time may exist (e.g. economic hardships, co-occurring undiagnosed depression) and may also affect one's perception of health. More research in this area is needed since residents of communities within Appalachian counties are almost twice as likely to report their general health as "poor or fair" than are community residents in other counties of the state whether or not they have a current health condition such as high blood pressure or a chronic disease such as cancer.

More white than black community residents in Appalachian counties report their health to be "poor or fair." Yet, only significant race/ethnic differences in rates of diabetes were found. White Appalachian community residents report good or excellent $\mathrm{PH}$ at the same rates as black community residents from other counties. A statistical trend indicates that more black residents in Appalachian counties perceived their general health as "good or excellent" compared to black residents in other counties $(P=.07)$, a finding that might reach the .05 level of significance with a larger sample. The difference in health perceptions between black respondents in communities in Appalachian counties and other counties has not been reported previously. "Relative deprivation" is a sociological term that refers to a gap in what one perceives that he or she is entitled to have, compared to what is the perceived 
norm [50, 51]. Perhaps black residents within communities in Appalachian counties have better perception of general health than black residents in other counties because they are comparing themselves to their white neighbors who have higher rates of chronic disease, poorer health perception and lower income status. More research is needed in this area because few studies of communities within Appalachia focus on non-European race/ethnic groups.

Cultural, geographical and other barriers to providing health care have been observed in Appalachia for decades, with significant improvements reported in many mountainous regions [52-55]. Yet, promoting wellness and the prevention of mortality and disease through proactive efforts of residents in Appalachian counties in VA and other Appalachian communities remains a challenge and higher mortality rates for many diseases continue to be reported $[56,57]$. The fact that having health insurance was not significantly related to health care utilization may be due to cultural factors and/or inability to make co-payments that are usually required by insurance companies that are not public. More research is needed in this area.

Since health perceptions have been associated with health behaviors, community residents in Appalachian counties may benefit from public health education and strategies in preventive medicine that are adapted or developed by the public health and health care professionals who live in the Appalachian region, with greater input from the residents in the communities who are most at risk. Since lower SES has been associated with lower participation rates in prevention efforts, more strategically focused materials are needed that respect the region's cultural beliefs, as well as the language of the Appalachian counties of the particular state. For example, "fair-tomiddling" is a unique phrase used in communities within Appalachian counties in VA to mean "about average" with regard to a question about how one is feeling, but responses may vary by Appalachian state [58].

This study found health-related differences between and among the residents within communities in the Appalachian counties compared to those in communities within other counties of the state. Further, there were both ethnic differences within the counties in Appalachia and between Appalachian counties and counties outside of the region. This study suggests that cultural and race/ethnic differences are both important when developing strategies to improve the health of the public within communities in specific state's counties in Appalachia in the US. However, more information is needed to inform such efforts.

Open Access This article is distributed under the terms of the Creative Commons Attribution Noncommercial License which permits any noncommercial use, distribution, and reproduction in any medium, provided the original author(s) and source are credited.

\section{References}

1. Appalachian Regional Commission: The Appalachian Region. (2009). Available at: http://www.arc.gov/appalachian_region/. Accessed August 17, 2010.

2. Cuoto, R. A., Simpson, N. K., Harris, G. (Eds.). (1994). Sowing seeds in the mountains: Community-based coalitions for cancer prevention and control. Bethesda, Md: Appalachia Leadership Initiative on Cancer, DCPC, National Cancer Institute; 14-28. NIH publication 94-377.

3. Campbell, J. C. (1921). The Southern highlander and his homeland. New York: Russell Sage Foundation.

4. Hackett, D. H. (1992). Albion's seed. Appalachian Journal, 19(2), 161-200.

5. Powell, D. R. (2007). Critical regionalism: Connecting politics and culture in the American landscape. North Carolina: The University of North Carolina Press.

6. Allen, S. D. (1995). More on the free black populations of the Southern Appalachian Mountains: Speculations on the North African connection. Journal of Black Studies, 25(6), 651-671.

7. Jordan, T. G., \& Kaups, M. (1989). The American backwoods frontier: An ethnic and ecological interpretation. Baltimore: John Hopkins University Press.

8. Newell-Withrow, C. (1997). A glance at Appalachia. Journal of Cultural Diversity, 4(4), 129-131.

9. Klotter, J. C. (1980). The Black South and White Appalachia. Journal of American History, 66(4), 832-849.

10. Weiner, D. (2000). Jewish women in the central Appalachian coal fields, 1890-1960: From breadwinners to community builders. American Jewish Archives, 52(1), 10-33.

11. Kennedy, N. B., \& Kennedy, R. V. (1997). The melungeons: The resurrection of a proud people: An untold story of ethnic cleansing in America. Macon: Mercer University Press.

12. Coyne, C. A., Demian-Popescu, C., \& Friend, D. (2006). Social and cultural factors influencing health in southern West Virginia: A qualitative study. Preventing Chronic Diseases Public Health Research, Practice Policy, 3(4), A124.

13. Caudill, H. M. (2001). Night comes to the cumberlands: A biography of a depressed area. Ashland Kentucky: Jesse Stuart Foundation.

14. Jesse, E. D., \& Reed, P. G. (2004). Effects of spirituality and psychosocial well-being on health risk behaviors in Appalachian pregnant women. Journal of Obstetric, Gynecologic, and Neonatal Nursing, 33(6), 739-747.

15. McMillan, S. J., Haley, E., Zollman-Huggler, P., Johnson, A. E., et al. (2007). Breast health education for working women in Appalachia: Insights from focus group research. Cancer Control: Journal of Moffitt Cancer Center, 14(3), 265-276.

16. Lobri-Posey, B. (2006). Middle-aged Appalachians living with diabetes mellitus: A family affair. Family and Community Health, 29(3), 214-220.

17. Deskins, S., Harris, C. V., Bradlyn, A. S., et al. (2006). Preventive care in Appalachia: Use of the theory of planned behavior to identify barriers to participation in cholesterol screenings among West Virginians. Journal of Rural Health, 22(4), 367-374.

18. Halverson, J. A., Barnett, E., \& Casper, M. (2002). Geographic disparities in heart disease and stroke mortality among black and white populations in the Appalachia region. Ethnicity and Disease, 12(4), S3-82-S3-91.

19. Kawachi, I., Daniels, N., \& Robinson, D. E. (2005). Health disparities by race and class: Why both matter. Health Affairs, 24(2), 343-352.

20. Braveman, P. (2006). Health disparities and health equity: Concepts and measurement. Annual Review of Public Health, 27, 167-194. 
21. Newell-Withrow, C. (2000). Health protecting and health promoting behaviors of African Americans living in Appalachia. Public Health Nursing, 17(5), 392-397.

22. Gamm, L., \& Hutchinson, L. (2003). Rural health priorities in America: Where you stand depends on where you sit. Journal of Rural Health, 19(3), 209-213.

23. U.S. Department of commerce economics and statistics administration. U.S. Census Bureau. (2009). Available at: http://fact finder.census.gov/home/saff/main.html?_lang=en. Accessed April 30, 2009.

24. Virginia Department of Health. Chronic Disease in Virginia: A Comprehensive Data Report, Division of Chronic Disease Prevention and Control. (2009) Available at: http://www:vahealth. org/cdp. Accessed April 25, 2009.

25. Huttlinger, K., Schaller-Ayers, J., \& Lawson, T. (2004). Health care in Appalachia: A population- based approach. Public Health Nursing, 21(2), 103-110.

26. Behringer, B., \& Friedell, G. H. (2006). Appalachia: Where place matters in health. Preventing Chronic Diseases Public Health Research, Practice and Policy, 3(4), A113.

27. Lengerich, E. J., Bohland, J. R., Brown, P. I., et al. (2006). Images of Appalachia. Preventing Chronic Diseases Public Health Research, Practice and Policy, 3(4), 1-3.

28. Hopenhayn, C., Bush, H., Christian, A., \& Shelton, B. J. (2005). Comparative analysis of invasive cervical cancer rates in three Appalachian states. Preventive Medicine, 41(5-6), 859-864.

29. Graduate Medication Education Consortium (GMEC), 2007. Report to the board Available at: http://www.uvawise.edu/gmec/ AnnualReport2007/annualreport2007.pdf. Accessed April 22, 2009.

30. Denham, S. A., Manoogian, M., \& Schuster, L. (2007). Managing family support and dietary routines: Type 2 diabetes in rural Appalachian families. Families, Systems, and Health, 25(1), 36-52.

31. Schaller-Ayers, J., Huttlinger, K., \& Lawson, T. (2001). Health care access in rural Appalachia podium presentation. Head for the hills: A retreat for primary care residents and faculty. Breaks, VA: Breaks Interstate Park.

32. McDowell, I., \& Newell, C. (1996). Measuring health: A guide to rating scales and questionnaires. New York: Oxford University Press.

33. Starfield, B. (2001). Basic concepts in population health and health care. Journal of Epidemiology and community health, 55(7), 452-458.

34. Cooper, J. K., \& Kohlman, T. (2001). Factors associated with health status of older Americans. Age and Ageing, 30(6), 495-501.

35. Duncan, G. E., Anton, S. D., Newton, R. L., \& Perri, M. G. (2003). Comparison of perceived health to physiological measures of health in black and white women. Preventive Medicine, 36(5), 624-628.

36. Lee, J. E., Lemyre, L., Turner, M. C., et al. (2008). Health risk perceptions as mediators of socioeconomic differentials in health behavior. Journal of Health Psychology, 13(8), 1082-1091.

37. Settersten, L. L., \& Lauver, D. R. (2004). Critical thinking, perceived health status, and participation in health behaviors. Nursing Research, 53(1), 11-18.

38. Steele, J. C., Patrick, J. H., Goins, R. T., \& Brown, D. K. (2005). Self-rated health among vulnerable older adults in rural Appalachia. Journal of Rural Health, 21(2), 182-186.

39. Pohar, S. L., Jones, C. A., Warren, S., et al. (2007). Health status and health care utilization of multiple sclerosis in Canada. The Canadian Journal of Neurological Sciences, 34(2), 167-174.
40. Rothman, A. J., \& Salovev, P. (1997). Shaping perceptions to motivate healthy behavior: The role of message framing. Psychological Bulletin, 121(1), 3-19.

41. Wheeler, J. R., Janz, N. K., \& Dodge, J. A. (2003). Can a disease self-management program reduce health care costs? The case of older women with heart disease. Medical Care, 41(6), 706-715.

42. Jacobsen, P. B., Lamonde, L. A., Honour, M., et al. (2004). Relation of family history of prostate cancer to perceived vulnerability and screening behavior. Psychooncology, 13(2), 80-85.

43. Shenson, D., Bolen, J., \& Adams, M. (2007). Receipt of preventive services by elders based on composity measures, 1997-2004. American Journal of Preventive Medicine, 32, 11-18.

44. Hughner, R. S., \& Kleine, S. S. (2004). Views of health in the lay sector: A compilation and review of how individuals think about health. Health (London), 8(4), 395-422.

45. Guterbock, T. M., Hartman, D. E., \& Kermer, D. (2007). Anthem TEACH Market Segmentation Study History and Methods Report. University of Virginia: Weldon Cooper Center for Public Service, Center for Survey Research.

46. McGuire, M. B. (1988). Ritual healing in suburban America. New Brunswick: Rutgers University Press.

47. Rizzo, L., Brick, J. M., \& Park, I. (2004). A minimally intrusive method for sampling persons in random digit dial surveys. Public Opinion Quarterly, 68(2), 267-274.

48. Standard Definitions: Final dispositions of case codes and outcome rates for RDD telephone surveys and in-person household surveys. (2009). Available at: www.aapor.org/uploads/standard_ definitions_07_08_final.pdf. Accessed April 3, 2009.

49. Mary Babb Randolph Cancer Center, Office for Social Environment and Health Research, Department of Community Medicine. (2008). Underlying socioeconomic factors influencing health disparities in the Appalachian Region. West Virginia University.

50. Merton, R. K. (1957). Social theory and social structure. New York: Free Press.

51. Crosby, F. (1976). A model of egoistical relative deprivation. Psychology Review, 83(2), 85-113.

52. Flannery, M. (1982). Appalachia health care: The struggle to reach remote areas. Appalachia, 15(4), 17-24.

53. Ward, A. J., Coffey Kluhsman, B., Lengerich, E. J., \& Piccinin, A. M. (2006). The impact of cancer coalitions on the dissemination of colorectal cancer materials to community organizations in rural Appalachia. Preventing Chronic Diseases Public Health Research, Practice and Policy, 3(2), A55.

54. Vanderpool, R. C., \& Coyne, C. A. (2006). Qualitative assessment of local distribution of screen for life mass medial material in Appalachia. Preventing Chronic Diseases Public Health Research, Practice and Policy, 3(2), A54.

55. Tessaro, I., Mangone, C., Parkar, I., \& Pawar, V. (2006). Knowledge, barriers, and predictors of colorectal cancer screening in an Appalachian church population. Preventing Chronic Diseases Public Health Research, Practice and Policy, 3(4), A123.

56. Armstrong, L. R., Thompson, T., Hall, H. I., et al. (2004). Colorectal carcinoma mortality among Appalachian men and women, 1969-1999. Cancer, 101(12), 2851-2858.

57. Health planning agency of Southwest Virginia, Inc. (2009). Available at: http://www.hpaswv.org. Accessed May 1, 2009.

58. Garber, A. (1976). Mountain-ese: Basic grammar for Appalachia. Radford: Commonwealth Press, Inc. 